\title{
LA PRODUCTIVIDAD TOTAL DE FACTORES INCORPORANDO VARIABLES AMBIENTALES: EL CASO PERUANO
}

\author{
Carlos Enrique Orihuela Romero ${ }^{1}$ José Luis Nolazco Cama²
}

Fecha de recepción: 01-10-12

Fecha de aceptación: 04-03-2013

\section{Resumen}

La Productividad Total de Factores (PTF) se obtiene a partir de una función de producción neoclásica la cual depende normalmente de los factores mano de obra y capital. Al ser obtenida como residual, la PTF mide la contribución al crecimiento del producto que no es explicada por ambos factores. Siguiendo a Tzouvelekas, Vouvaki y Xepapadeas (2007), el presente estudio propone incorporar un factor ambiental (medido como emisiones de $\mathrm{CO}_{2}$ ) y un factor humano (capital humano) a la función de producción agregada del Perú a fin de obtener un PTF más apropiado. Usando series de tiempo para el periodo 1960-2009, se encontró que la variable ambiental resultó significativa y por ende, su omisión podría sobreestimar la típica PTF. Asimismo, se demuestra que el capital humano no explica el producto, lo cual va en línea con lo encontrado por Carranza, Fernández-Baca y Morón (2003) y Yamada (2006) para el caso peruano.

Palabras clave: Solow, Productividad Total de Factores, crecimiento, ambiente.

\section{Clasificación JEL: O44,O47, Q5}

Magister Economía de los Recursos Naturales y del Medio Ambiente (Universidad de Concepción - Chile). Docente e investigador (UNALM). Dirección postal: Jr. Pirandello 105, Dpto 102 (Lima, Perú). Teléfono: (511) 6147800 anexo: 239; e-mail: corihuela@lamolina.edu.pe

\section{Abstract}

Total Factor Productivity (TFP) is obtained from a neoclassical production function which usually depends on the factors labor and capital. To be obtained as a residual, TFP measures the contribution to output growth that is not explained by two factors. According Tzouvelekas, Vouvaki and Xepapadeas (2007), this study proposes to incorporate an environmental factor (measured as $\mathrm{CO}_{2}$ emissions) and human capital to the aggregate production function of Peru in order to obtain a PTF more appropriate. Using time series for the period 1960-2009, it was found that the environmental variable was significant and hence their omission could overestimate the typical PTF. It also shows that human capital does not explain the product, which is in line with findings by Carranza, Fernández-Baca and Morón (2003) and Yamada (2006) for Peru.

Key words: Solow, Total Factor Productivity, growth, environment.

\section{JEL Classification: O44, O47, Q5}

2 Economista (UNALM-Perú). Consultor del Programa de Desarrollo Rural Sostenible (PDRS) de la GTZ. Asistente de docencia (UNALM-Perú). Dirección postal: Calle Helsinski 363, Ate-Vitarte. Teléfono: (511) 3511463; e-mail: 20050941@ lamolina.edu.pe 


\section{INTRODUCCIÓN}

En la década de los cincuenta, Solow (1957) estableció las bases de la teoría del crecimiento económico del producto, el cual -conforme a su planteamiento- es explicado no solo por la contribución de los factores clásicos de producción, tales como capital y mano de obra, sino también por el componente denominado Productividad Total de Factores (PTF), el cual mide la fracción del producto que no es explicada por tales factores.

Esta teoría ha sido cuestionada por la omisión de la variable ambiental (Georgescu-Roegen, 1975), lo cual podría sesgar los resultados al no incluir, por ejemplo, los costos de contaminación en el crecimiento económico (Brock, 1973). Ya por la década de los ochenta, esta teoría entró en transición una vez que aparecieron los modelos de crecimiento endógeno los cuales se alejan del planteamiento neoclásico al suponer una tasa de crecimiento endógena que permite incorporar las preferencias, la tecnología y las políticas regulatorias a los procesos de crecimiento económico (Romer, 1987; Grossman y Helpman, 1991; Aghion y Howitt, 1992).

Posteriormente, la discusión entre el crecimiento económico y el deterioro ambiental tomó fuerza a fines de los noventa probando la hipótesis de la Curva Ambiental de Kuznets que, en líneas generales, propone la existencia de una relación en forma de U-invertida entre el deterioro medio ambiental y el crecimiento económico (Stern, Common y Barbier, 1996; Ekins, 1997; De Bruyn et al., 1998; Harbaugh y Levinson, 2002). Sin em- bargo, estos estudios no estuvieron exentos de críticas en la estimación econométrica tales como presencia de heterogeneidad, simultaneidad, sesgo de variables omitidas y cointegración (Stern, 2004).

En la actualidad, ha resurgido el análisis de la contabilidad del crecimiento verde en los modelos neoclásicos estudiados en la década de los setenta ${ }^{3}$, y en la cual se analiza la incorporación de la variable ambiental en la explicación del producto, para así cuantificar una verdadera PTF (Xepapadeas 2003, 2005; Kalaitzidakis, Mamuneas y Stengos, 2006; Tzouvelekas, Vouvaki y Xepapadeas, 2007). La premisa es que es que existirá un sesgo al omitir la variable ambiental pues la PTF será explicada solo por cambios tecnológicos, desarrollo del capital humano, estabilidad política y macroeconómica, solvencia del sistema financiero, entre otros, excepto por la degradación ambiental.

El primer objetivo de este trabajo es de tipo metodológico. Se plantea la necesidad de incorporar en el producto no solo el capital artificial, mano de obra y capital humano, sino también la variable ambiental. Se propone que este último factor debe incorporarse en cualquier estudio empírico de crecimiento.

El segundo objetivo es calcular una PTF más apropiada para el Perú, lo que puede generar mejoras en las conclusiones y/o medidas de políticas públicas que permitan lograr el objetivo estratégico de la alta competitivi-

3 Para los primeros estudios sobre el análisis de la contaminación ambiental en los modelos de crecimiento en estilo neoclásico, véase, por ejemplo Keeler, Spence Zeckhauser (1971), Brock (1973), Gruver (1976) y Becker (1982). 
dad con mejores niveles de empleo, basada en un estructura productiva diversificada, competitiva, sostenible y con alto valor agregado. Esta hipótesis se prueba a través de modelos no lineales en parámetros para series de tiempo durante el periodo 19602009 corroborando previamente -a través de la metodología de Granger (1988)- que las variables regresoras explican al producto $y$ no lo contrario.

El resto del documento está estructurado de la siguiente manera: en la sección 2 , se realizará una revisión de literatura de la medición de la PTF y la inclusión de la variable ambiental en la explicación del producto; en la sección 3 se analiza la PTF a partir del modelo de Solow y se deriva una PTFA a partir de la inclusión del factor ambiental en tal modelo; en la sección 4 se señalan las fuentes de los datos y los modelos a desarrollar en las estimaciones econométricas; mientras que en la sección 5 se explicará los resultados obtenidos y el valor de la PFTA, evaluando previamente la relación existente entre las regresoras y el producto. Finalmente, las conclusiones y recomendaciones se presentan en la sección 6 .

\section{REVISIÓN DE LITERATURA}

Son abundantes los estudios que han calculado la PTF tradicional para las economías de diversos países e incluso para los sectores que la componen. Sin embargo, la literatura empírica que estima la PTF incluyendo la variable ambiental es reciente.
Xepapadeas (2003, 2005) ofreció un análisis de la relación entre el crecimiento económico y la degradación ambiental en la función de producción neoclásica de Solow. Dicho autor demuestra la posible dependencia de las emisiones de $\mathrm{CO}_{2}$ y el producto.

Kalaitzidakis, Mamuneas y Stengos (2006) demostraron empíricamente el efecto de la contaminación -medido por las emisiones de $\mathrm{CO}_{2}$ - en el crecimiento económico para los países industrializados. Usando un modelo semiparamétrico para el periodo 1981-1998, los autores encontraron una relación no lineal significativa entre el crecimiento de la PTF y las emisiones de $\mathrm{CO}_{2}$, la cual varía en función del nivel de contaminación de un país y el nivel de capital humano.

Tzouvelekas, Vouvaki y Xepapadeas, (2007) también incorporaron las emisiones de $\mathrm{CO}_{2}$ como proxy de la degradación ambiental en el producto y analizaron su contribución en la medición de la PTF mediante un modelo de datos panel para 23 países de la OCDE. Los resultados sugieren que tales emisiones tienen una contribución estadísticamente significativa para la PTF. Sin embargo, una particularidad de estos últimos estudios es que omiten la prueba de causalidad entre las emisiones de $\mathrm{CO}_{2}$ y el producto. Tener en consideración este último análisis, permitirá evaluar la viabilidad de incluir la variable ambiental como factor del producto.

Justamente, Coondoo y Dinda (2002) cuestionan los estudios que evalúan la relación entre el producto y nivel de contaminación. Utilizando un modelo de datos panel, los autores demostraron, a través del test de causalidad 
de Granger, que en países desarrollados de Norte América y Europa Occidental la causalidad va desde las emisiones de $\mathrm{CO}_{2}$ hacia el producto, mientras para los grupos de países en desarrollo como América del Sur, Centroamérica y Oceanía la causalidad es de manera inversa. Incluso, para Asia y África encontraron que esta relación es bidireccional, aunque la heterogeneidad entre los grupos de países pudo ocasionar sesgos en los resultados.

Por esa razón, Menyah y Wolde-Rufael (2010) evaluaron la relación causal en el largo plazo del crecimiento económico, las emisiones contaminantes y el consumo de energía para el sur de África durante el período 1965-2006, aplicando la metodología de cointegración de Johansen. Mediante la prueba de causalidad de Granger, los autores demostraron una relación unidireccional tanto de las emisiones de $\mathrm{CO}_{2}$ y del consumo de energía hacia el crecimiento económico.

Para el caso peruano, Vega-Centeno (1997), Seminario y Beltrán (1998), Carranza, Fernández-Baca y Morón (2003), IPE (2003), estimaron la PTF sin factor ambiental aunque en algún caso (Jiménez, 2011) también incluyeron el capital humano. Sobre esta última variable, vale destacar que no hay estudios rigurosos sobre su inclusión en la función de producción agregada peruana debido a la ausencia de información confiable de la calidad de educación (Carranza, Fernández-Baca y Morón, 2003, Yamada, 2006; Morón, 2007; Jiménez, 2011).

En síntesis, dada la deficiencia en el cálculo de la PTF para el caso peruano, a continuación se propone incorporar la variable ambiental como input en el producto evaluando previamente, la causalidad entre estas. Posteriormente, se calculará una nueva PTF para los fines ya mencionados.

\section{EL MODELO}

\subsection{Modelo de Solow sin medio ambiente ${ }^{4}$}

En el modelo clásico de crecimiento de Solow tradicional, sin consideraciones ambientales, se tiene:

$$
Y=F(K, E)=F(K, A L)
$$

En términos de crecimiento, la expresión (1) se puede escribir de la siguiente manera:

$$
\frac{\dot{Y}}{Y}=S_{K}\left(\frac{\dot{\mathrm{K}}}{K}\right)+S_{L}\left(\frac{\dot{\mathrm{A}}}{A}\right)+S_{L}\left(\frac{\dot{L}}{L}\right)
$$

Donde $Y$ es el producto agregado, $K$ es el capital físico, $E=A L$ es el trabajo efectivo, siendo $L$ la mano de obra (input) y $A$ es un parámetro que incrementa el cambio técnico. Asimismo, $S_{K}$ y $S_{L}$ son las participaciones del capital y del trabajo en el producto. La PTF se define como:

$$
g_{S}=S_{L}\left(\frac{\dot{A}}{A}\right)=\frac{\dot{Y}}{Y}-S_{K}\left(\frac{\dot{K}}{K}\right)-S_{L}\left(\frac{\dot{L}}{L}\right)
$$

Bajo retornos constantes a escala, $S_{K}+S_{L}=$ 1, y por tanto la expresión (3) se convierte en:

$$
g_{S}=S_{L}\left(\frac{\dot{A}}{A}\right)=\frac{\dot{y}}{y}-S_{K}\left(\frac{\dot{k}}{k}\right)
$$

4 
Donde $\frac{\dot{y}}{y}$ es el ratio de crecimiento del producto por trabajador $\left(y=\frac{Y}{L}\right)$ y $\frac{\dot{k}}{k}$ es el ratio del crecimiento del capital por trabajador $(\mathrm{k}=K / L)$. Por lo tanto, la PTF está dada por $S_{L}\left(\frac{\dot{A}}{A}\right)$, donde el ratio del trabajo exógeno que aumenta el cambio técnico $x=\frac{\mathrm{A}}{A}$ puede ser directamente determinado.

\subsection{Modelo de Solow}

\section{Ambiental $^{5}$}

Usando las ideas de Denison (1962), Dasgupta y Mäler (2000) y Tzouvelekas, Vouvaki y Xepapadeas, (2007), quienes incorporaron el capital humano y la variable ambiental como inputs del producto, la nueva función de producción neoclásica estándar será:

$$
Y=F(K, E, H, X)
$$

Donde en adición al $K$ y $E, H$ es stock de capital humano, $X=B Z$ es la multiplicación de la variable ambiental $Z$ y el ahorro o aumento en el cambio técnico $B$ (también denominado residual ambiental). Diferenciando (5) respecto al tiempo, la ecuación de crecimiento será:

$$
\bar{Y}=S_{K}\left(\frac{\dot{K}}{K}\right)+S_{H}\left(\frac{\dot{H}}{H}\right)+S_{L}\left(\frac{\dot{A}}{A}\right)+S_{L}\left(\frac{\dot{L}}{L}\right)+S_{Z}\left(\frac{\dot{B}}{B}\right)+S_{Z}\left(\frac{\dot{Z}}{\bar{Z}}\right)
$$

Donde $S_{j}(j=K, L, H, Z)$ representa la participación de los factores en el producto. Por lo tanto, de la expresión (6) la PTF ajustada (PTFA) se define como $\gamma$ :

$\gamma=S_{L}\left(\frac{\dot{A}}{\bar{A}}\right)+S_{Z}\left(\frac{\dot{B}}{B}\right)=\frac{\dot{Y}}{\bar{Y}}-S_{K}\left(\frac{\dot{K}}{\bar{K}}\right)-S_{H}\left(\frac{\dot{H}}{H}\right)-S_{L}\left(\frac{\dot{L}}{L}\right)-S_{Z}\left(\frac{\dot{Z}}{Z}\right)$
Donde bajo retornos constantes a escala: $S_{K}+S_{H}+S_{L}+S_{Z}=1$ Asimismo, la expresión (7) se puede expresar como tasa de crecimiento:

$$
\gamma=\frac{\dot{Y}}{Y}-S_{K}\left(\frac{\dot{k}}{k}\right)-S_{H}\left(\frac{\dot{h}}{h}\right)-S_{Z}\left(\frac{\dot{Z}}{Z}\right)
$$

A diferencia de (3) ó (4), las expresiones (7) u (8)incluyen la variable ambiental $\left(s_{\downarrow} Z Z^{*} / Z\right)$ y el capital humano $S_{H}\left(\frac{\dot{H}}{H}\right)$, lo cual indica que existen dos recursos más que generan crecimiento de la producción, además del stock de capital artificial y la mano de obra. Por lo tanto, $\mathrm{Z}$ y $\mathrm{H}$ deberían ser consideradas con el fin de obtener una estimación ajustada de la PTF.

\section{METODOLOGÍA}

\subsection{Los Datos}

Esta sección proporciona las fuentes de las variables que sirvieron para estimar econométricamente la PTFA durante el periodo 1960-2009. Para la estimación de la variable dependiente, el producto, se utilizó la data del producto interno bruto (PIB), cuya información fue obtenida del Banco Central de Reserva del Perú. Las variables regresoras fueron: stock de capital artificial (K), stock de capital humano $(\mathrm{H})$ y como proxy de la degradación de los recursos naturales, se utilizó las emisiones de $\mathrm{CO}_{2}(\mathrm{Z})$.

El uso de esta última variable, para el caso peruano, obedeció principalmente a tres razones. En primer lugar, de todos los contaminantes, las emisiones de $\mathrm{CO}_{2}$ son las más representativas: en 1994 concentraron el 97\% del total de emisiones de efecto invernadero 
(MINEM, 2010). En segundo lugar, dicha variable se genera a partir de la contaminación por sectores económicos tales como transporte, pesca, industria, agropecuario, minería y otros que de una u otra forma, generan degradación de los recursos naturales. Por último, las emisiones de $\mathrm{CO}_{2}$ han sido utilizadas en estudios de crecimiento que incluyen la variable ambiental tales como Kalaitzidakis, Mamuneas y Stengos, (2006) y Tzouvelekas, Vouvaki y Xepapadeas (2007).

La información de K se obtuvo de Seminario et al. (2008) para el periodo 1960-2007. Para el periodo restante (2008-2009) se estimó mediante extrapolación lineal simple. $L$ a variable $H$ fue estimada usando la expresión $\mathrm{H}=\mathrm{PEA}\left(\mathrm{e}^{\alpha \theta}\right)$ donde PEA es la población económicamente activa, que en el Perú está comprendida entre los 15 y los 65 años; $\theta$ es el número de años de logro educativo mientras que a corresponde a la tasa de retorno de la educación.

Para el periodo 1984-2004 la información de a fue obtenida de Yamada (2007), mientras que para los periodos 1960-1983 y 2005-2009 se utilizaron los valores de los años 1984 y 2004, respectivamente. La información de $\theta$ fue obtenida de Barro y Lee (2000) quienes ofrecen estimaciones por quinquenio. La información de la PEA se obtuvo de INEI (2010a) para el periodo 1970-2009, mientras que para el periodo 1960-1969 se estimó mediante extrapolación lineal simple.

La información de Z fue obtenida de INEI (2010b) para todo el periodo 1985-2009. Para el periodo restante, se utilizó la infor- mación del World Bank (2011). Las series de $\mathrm{K}, \mathrm{H}$ y $\mathrm{Z}$ fueron divididas entre la PEA (L). Las series monetarias fueron convertidas a soles constantes del año 1994, utilizando el deflactor implícito del PIB.

\subsection{Modelos para la estimación de la PTFA}

Sea la especificación Cobb-Douglas estándar, incluyendo las emisiones de $\mathrm{CO}_{2}$ y el capital humano:

$$
Y=A K^{S_{K}} H^{S_{H}}(A L)^{S_{L}}(B Z)^{S_{Z}}
$$

La función de producción agregada (9) expresada en términos por trabajador $(P E A=L)$ sería:

$$
\frac{Y}{L}=\left(\frac{K}{L}\right)^{S_{K}}\left(\frac{H}{L}\right)^{S_{H}}\left(\frac{A L}{L}\right)^{S_{L}}\left(\frac{B Z}{L}\right)^{S_{Z}}
$$

Y, sabiendo que $A=e^{b_{1} t}$ y $B=e^{b_{2} t}$, donde $b_{1}$ y $b_{2}$ son parámetros que representan la tasa de crecimiento constante del cambio técnico de la mano de obra y emisiones de $\mathrm{CO}_{2}$, respectivamente, se tiene:

$$
y=k^{S_{K}} h^{S_{H}} Z^{S_{Z}} e^{\left(b_{1} S_{L}+s_{Z} b_{2}\right) t}
$$

Donde: $y=\frac{Y}{L}, k=\frac{K}{L}, h=\frac{H}{L}$ y $z=\frac{Z}{L}$. Linealizando (11), se obtienen las elasticidades de cada input:

$$
\begin{gathered}
\ln y=\left(s_{L} b_{1}+s_{Z} b_{2}\right) t+s_{K} \ln k+s_{H} \ln h+s_{Z} \ln z \\
s_{Z}=1-s_{k}-s_{H}-s_{L}
\end{gathered}
$$

Donde: PTFA $=\left(s_{L} b_{1}+s_{Z} b_{2}\right)$. Asimismo, si a 
(12) se impone $a_{2}=0$, se obtiene una función de producción con emisiones de $\mathrm{CO}_{2}$ pero sin capital humano:

$$
\ln y=\left(s_{L} b_{1}+s_{Z} b_{2}\right) t+s_{K} \ln k+s_{Z} \ln z
$$

Por otro lado, si $S_{Z}=0$ entonces en (12) se tiene a la función de producción agregada tradicional con capital humano sin emisiones:

$$
\ln y=\left(s_{L} b_{1}\right) t+s_{K} \ln k+s_{H} \ln h
$$

Donde: PTF $=\left(s_{L} b_{1}\right)$. Adicionalmente, si $s_{H}=s_{Z}=0$, (12) se transforma en la función de producción neoclásica estándar:

$$
\ln y=\left(s_{L} b_{1}\right) t+s_{K} \ln k
$$

Cada una de estas especificaciones (12), (13), (14) y (15), pueden ser asociadas a distintas ecuaciones de crecimiento. Específicamente en (12), que es la ecuación más general, se tiene:

$$
\frac{\dot{Y}}{Y}=\gamma+S_{K}\left(\frac{\dot{k}}{k}\right)-S_{H}\left(\frac{\dot{h}}{h}\right)-S_{Z}\left(\frac{\dot{z}}{z}\right)
$$

Donde la PTFA: $\quad \gamma=s_{L} b_{1}+s_{Z} b_{2}$

Por lo tanto, la PTFA puede ser estimada econométricamente, incluyendo una tendencia, en las ecuaciones (12)-(15), o una constante en la ecuación (16). Sin embargo, esta última ecuación no será estimada, pues tiene la desventaja de no poder separar las contribuciones del cambio técnico asociados a la mano de obra $\left(b_{\downarrow} 1\right)$ y las emisiones de $\mathrm{CO}_{2}\left(b_{\downarrow} 2\right)$. Asimismo, las estimaciones usando una función de producción en primeras diferencias (según la aproximación $x / x \cong \ln x_{\downarrow} t-\ln x_{\downarrow}(t-1)$ podrían presentar problemas asociados con la estacionariedad de las variables en niveles.

Teniendo en cuenta la dependencia de los parámetros asociados a cada factor de la producción y la necesidad de estimar $b_{1}$ y $b_{2}$ (parámetros irrestrictos), es claro que las ecuaciones (12)-(15) no pueden ser estimadas vía una tendencia simple. Para solucionar este problema, haciendo $s_{Z}+s_{K}+s_{H}+s_{L}=1$, se plantean los siguientes modelos no lineales en parámetros:

\section{PTFA1:}

$\ln \left(\frac{y}{z}\right)=\left(s_{L} b_{1}+\left[1-s_{K}-s_{H}-s_{L}\right] b_{2}\right) t+s_{K} \ln \left(\frac{k}{z}\right)+s_{H} \ln \left(\frac{h}{z}\right)-\left[s_{L}\right] \ln z$

Donde la PTFA es igual a:

$s_{L} b_{1}+\left[1-s_{K}-s_{H}-s_{L}\right] b^{2}=s_{L} b_{1}+s_{Z} b_{2}(17)$ PTFA1:

$\ln \left(\frac{y}{z}\right)=\left(s_{L} b_{1}+\left[1-s_{K}-s_{L}\right] b_{2}\right) t+s_{K} \ln \left(\frac{k}{z}\right)-\left[s_{L}\right] \ln z \quad$ (18)

Siendo la PTFA igual a: $s_{L} b_{1}+\left[1-s_{K}-s_{L}\right] b_{2}=s_{L} b_{1}+s_{Z} b_{2}$ y $s_{H}=0$. Asimismo, a fin de comparar las variantes para la estimación del PTFA, se plantea los siguientes modelos:

PTF1:

$\ln y=\left(\left[1-s_{K}-s_{H}\right] b_{1}\right) t+s_{K} \ln k+s_{H} \ln h$ (19)

Donde la PTF es igual a: 


$$
\left[1-s_{K}-s_{H}\right] b_{1}=s_{L} b_{1} \text { y } s_{Z}=0
$$

PTFA2: $\ln y=\left(\left[1-s_{K}\right] b_{1}\right) t+s_{K} \ln k$

Donde la PTF es igual a:

$$
\left[1-s_{K}\right] b_{1}=s_{L} b_{1} \text { y } s_{H}=s_{Z}=0
$$

Para evitar el problema de endogeneidad en los modelos (17)-(20) asociado con los inputs e inconsistencia en los estimadores de la función de producción, se estima en PTFA1 - PTFA2 y PTF1 - PTF2 bajo retornos constantes a escala, ya que en este caso los estimadores MCO son consistentes para el caso de una función de producción de tipo Cobb-Douglas (Mundlak, 1996).

\section{RESULTADOS}

\subsection{Causalidad entre el producto y variables regresoras}

Antes de proceder a la estimación de la PTFA, es necesario explicar el tipo de causalidad existente entre las variables regresoras y el producto. Siguiendo la metodología de Granger (1988) se propone probar el test de causalidad para tres sub periodos, 1960-1990, 1960-1999 y 1993-2009, así como para todo el horizonte de análisis, 1960-2009.

El primer periodo (1960-1990) fue escogido para compararlo con los resultados de Coondoo y Dinda (2002) quienes obtuvieron una relación unidireccional que va desde el pro- ducto hacia las emisiones de $\mathrm{CO}_{2}$ para América del Sur.

El segundo periodo (1960-1999) corresponde al estudiado por Carranza, Fernández-Baca y Morón (2003) para el caso peruano, de manera que su resultado pueda ser comparado con los obtenidos en este estudio. Durante una parte de este periodo (1970-1992), el Perú experimentó profundos cambios macroeconómicos (hiperinflación, altos niveles de desempleo, recesión), incluso el flagelo del terrorismo, de manera que los resultados podrían estar severamente distorsionados por estos eventos.

En el tercer periodo (1993-2009), la economía peruana inició un proceso de recuperación, emprendiéndose profundas reformas estructurales que contribuyeron a un sostenido crecimiento económico.

Conforme a la Tabla 1, solo las variables regresoras convencionales $(\mathrm{K}$ y $\mathrm{L}$ ) presentaron la dirección de causalidad esperada: ambas inciden sobre el producto, y no lo contrario para los cuatro periodos de análisis. Sin embargo, las variables regresoras no convencionales $(\mathrm{H}$ y Z) tuvieron un comportamiento ambiguo dependiendo del periodo.

Los resultados para todos los periodos indican que el producto no explica el capital humano y tampoco de manera inversa. Lo anterior coincide a lo obtenido para la PTF peruana por Carranza, Fernández-Baca y Morón (2003), Yamada (2006) y Jiménez (2011), quienes tampoco encuentran alguna relación entre el capital humano y el producto, adu- 
ciendo que esto se debe a la ausencia de información confiable en la construcción de dicho factor. Por tanto, no debería estimarse la ecuación PTFA1 para ningún periodo ya que se estaría incluyendo una variable espuria en la regresión.
Para los periodos $1960-1990$ y $1960-1999$, la ecuación correcta a estimar seria PTF2. En el primer periodo, el producto explica las emisiones de $\mathrm{CO}_{2}$ y no de manera inversa, lo cual coincide a lo obtenido por Coondoo y Dinda (2002) y por lo tanto, no sería correcto incluir las emisiones de $\mathrm{CO}_{2}$ como un input en dicho

Tabla 1. Causalidad de Granger ${ }^{1 /}$

\begin{tabular}{|c|c|c|c|c|c|c|c|c|}
\hline \multirow[b]{2}{*}{ Hipótesis Nula } & \multicolumn{2}{|c|}{$1960-1990$} & \multicolumn{2}{|c|}{ 1960-1999 } & \multicolumn{2}{|c|}{ 1993-2009 } & \multicolumn{2}{|c|}{ 1960-2009 } \\
\hline & $\begin{array}{l}\text { Prob. } \\
\text { Chi2 }\end{array}$ & $\begin{array}{c}\text { Prob. } \\
\text { F }\end{array}$ & $\begin{array}{l}\text { Prob. } \\
\text { Chi2 }\end{array}$ & $\begin{array}{c}\text { Prob. } \\
\text { F }\end{array}$ & $\begin{array}{l}\text { Prob. } \\
\text { Chi2 }\end{array}$ & $\begin{array}{c}\text { Prob. } \\
\text { F }\end{array}$ & $\begin{array}{c}\text { Prob. } \\
\text { Chi2 }\end{array}$ & $\begin{array}{c}\text { Prob. } \\
\text { F }\end{array}$ \\
\hline $\ln y$ no causa a lo Granger a $\ln z$ & 0.01 & 0.00 & 0.17 & 0.19 & 0.48 & 0.49 & 0.06 & 0.07 \\
\hline $\ln z$ no causa a lo Granger a $\ln y$ & 0.24 & 0.20 & 0.10 & 0.12 & 0.00 & 0.02 & 0.01 & 0.01 \\
\hline Rezago Óptimo del VAR & \multicolumn{2}{|c|}{3} & \multicolumn{2}{|c|}{2} & \multicolumn{2}{|c|}{1} & \multicolumn{2}{|c|}{2} \\
\hline $\ln y$ no causa a lo Granger a $\ln k$ & 0.81 & 0.81 & 0.29 & 0.30 & 0.92 & 0.93 & 0.13 & 0.14 \\
\hline $\ln k$ no causa a lo Granger a $\ln y$ & 0.04 & 0.06 & 0.02 & 0.03 & 0.07 & 0.10 & 0.00 & 0.00 \\
\hline Rezago Óptimo del VAR & \multicolumn{2}{|c|}{2} & \multicolumn{2}{|c|}{2} & \multicolumn{2}{|c|}{1} & \multicolumn{2}{|c|}{1} \\
\hline $\ln y$ no causa a lo Granger a $\ln h$ & 0.31 & 0.33 & 0.09 & 0.13 & 0.69 & 0.69 & 0.95 & 0.95 \\
\hline $\ln h$ no causa a lo Granger a $\ln y$ & 0.93 & 0.93 & 0.93 & 0.93 & 0.98 & 0.99 & 0.55 & 0.54 \\
\hline Rezago Óptimo del VAR & \multicolumn{2}{|c|}{1} & \multicolumn{2}{|c|}{3} & \multicolumn{2}{|c|}{1} & \\
\hline Resultado & \multicolumn{2}{|c|}{ PTF2 } & \multicolumn{2}{|c|}{ PTF2 } & \multicolumn{2}{|c|}{ PTFA2 } & \multicolumn{2}{|c|}{ PTFA2 } \\
\hline
\end{tabular}

"Las variables se testearon en primeras diferencias para que sean estacionarias. Asimismo, todas las variables están en términos por trabajador (L).

Fuente: Elaboración propia.

periodo ya que ocasionaría endogeneidad en las estimaciones. Para el segundo periodo, las emisiones de $\mathrm{CO}_{2}$ no explican el producto y tampoco de manera inversa. Sin embargo, durante los periodos 1993-2009 y 1960-2009, los resultados sugieren que el stock de capital artificial y las emisiones de $\mathrm{CO}_{2}$ explican el producto y no de manera inversa para un nivel de significancia del 10\%.Considerando dichas variables como inputs, la ecuación correcta a estimar en ambos casos sería PTFA2.

El efecto que las emisiones de $\mathrm{CO}_{2}$ incidan sobre el producto o que ocurra lo contrario depende- ría de dos situaciones: i) la eficiencia en el uso de la energía (diferentes cantidades de energía para producir un mismo producto) y ii) la concentración de la actividad económica en sectores más o menos intensivos en el uso de energía.

Durante el periodo 1960-1990, la incidencia del PIB sobre las emisiones de $\mathrm{CO}_{2}(Z)$ puede explicarse por los efectos de la deforestación y el cambio de uso en el suelo (CONAM, 2001), eventos que contribuyeron con más del $47 \%$ de las emisiones totales a nivel nacional en el año 2000. El resto está conformado por la generación eléctrica e hidrocarburos y por 
el consumo de energía que, adicionalmente, abarca los sectores industria comercial y doméstico (MINAM, 2010).

El aumento poblacional, los ciclos económicos y otros eventos socioeconómicos, conllevaron a una creciente migración hacia la amazonia, cambiando el uso del suelo para fines agrícolas, aún cuando esta presión ha sido decreciente en algunos periodos. Por el lado de la industria, la eficiencia energética fue baja en este periodo (Gráfico 1) debido posiblemente al débil sistema regulatorio imperante en la industria peruana en aquella época.

De otro lado, a inicios de los noventa se emprendieron profundas reformas estructurales en todo el país, con el fin de reactivar la economía. Estas reformas abarcaron no solo el sector energético sino también los sectores transportes, saneamiento y telecomunicaciones. Posteriormente, en el año 2004 se inició el Proyecto Camisea el cual consiste en la extracción y distribución de las cuantiosas reservas de gas natural y líquidos (LGN), cuya importancia se reflejó en el incremento del PIB en 1\%, modificando así la matriz energética, lo que significó un decrecimiento sostenible de la intensidad energética (MINEM, 2010). Todo esto explicaría, en parte, la incidencia de las emisiones de $\mathrm{CO}_{2}$ sobre el producto durante el periodo 1990-2009.

En resumen, se corrobora que la causalidad entre la variable dependiente y la variable ambiental difiere entre el periodo 1960-2009 y el subperiodo 1960-1990. Por lo tanto, es importante tener en cuenta que las estimaciones pueden estar sesgadas si no se realiza un previo análisis de causalidad.

\subsection{Estimación Econométrica de la PTFAz}

Una vez determinada la relación de causalidad para evaluar qué ecuación debería estimarse para diferentes periodos de tiempo, se plantea la medición de la PTFA. A continuación, se determina y compara los resultados de la PTFA $\left(s_{L} b_{1}+s_{\downarrow} Z b_{\downarrow} 2\right)$ y PTF $\left(s_{L} b_{1}\right)$ para los periodos 1960-1999, 1993-2009 y 19602009, obteniendo incluso las contribuciones del cambio técnico asociados a la mano de obra $\left(b_{\downarrow} 1\right)$ y a las emisiones de $\mathrm{CO}_{2}\left(b_{\downarrow} 2\right)$. Cabe añadir que para obtener dichos resultados, se plantea un modelo no lineal en parámetros cuya ventaja radica en obtener la participación de cada factor en el producto, mas aún, si este explicado por más de dos inputs.

En primer lugar, es importante también mencionar que para los diferentes periodos algunas variables son no significativas individualmente debido a la multicolinealidad entre el stock de capital artificial, stock de capital humano y la mano de obra (Anexo 3). Sin embargo, a pesar de este problema, los estimadores siguen siendo insesgados. Por ello, el presente estudio evita estimar la PTFA por décadas, ya que ante problemas de multicolinealidad los parámetros son muy sensibles y pueden carecer de una medición adecuada en los estimadores y dela PTFA (Green, 1990).

En segundo lugar, la mayoría de modelos es- 
Figura 1. PIB y Emisiones por trabajador durante el periodo 1960-2009

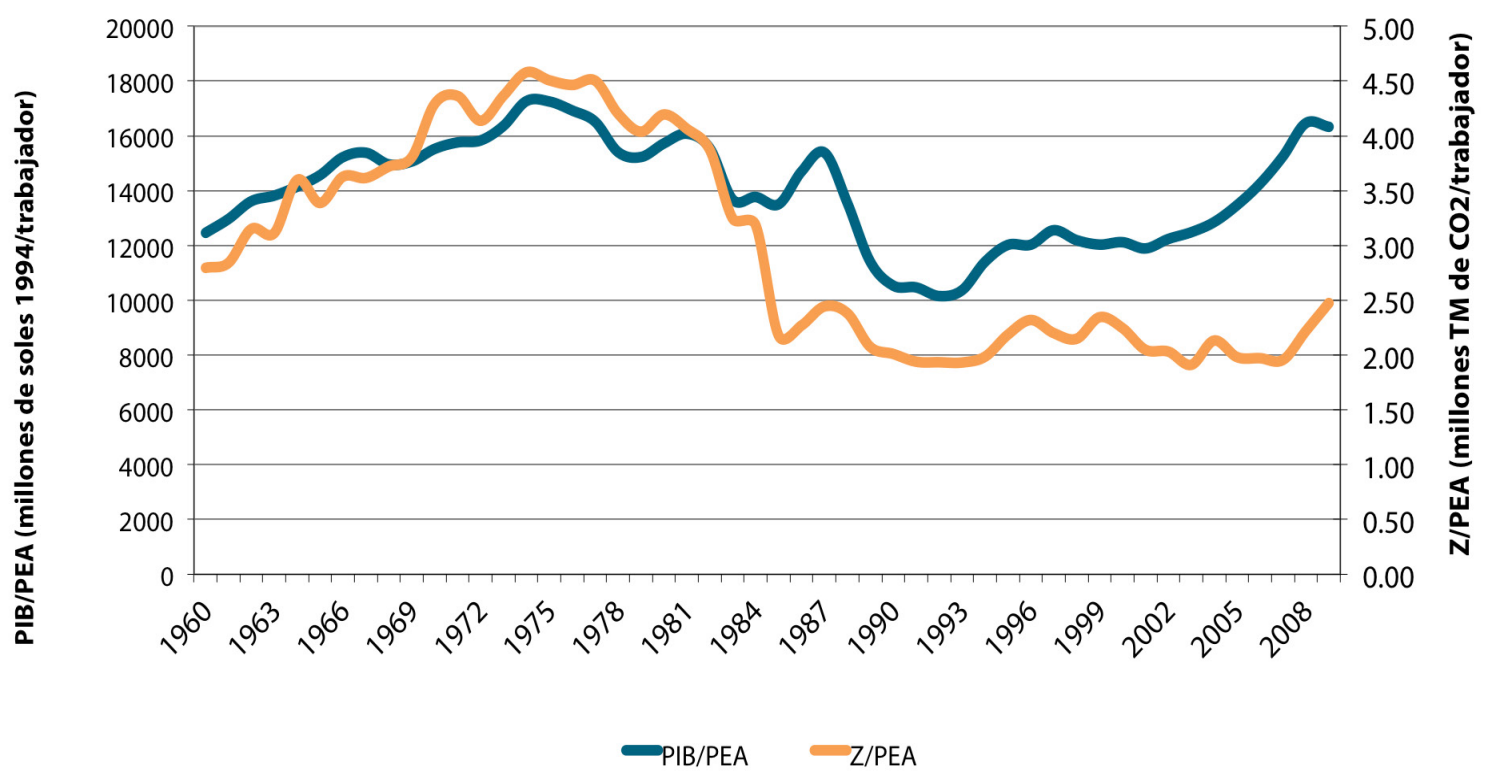

Fuente: Elaboración propia en base a BCRP (2011) e INEI (2010).

timados para los diferentes periodos fueron corregidos de problemas de heteroscedasticidad y autocorrelación utilizando el estimador consistente de Newey-West (HAC). Asimismo, todos los estimadores resultaron globalmente significativos incluso al 1\% (Anexo 4).

Según lo obtenido por el test de causalidad durante los períodos1960-1999, 1993-2009 y 1960-2009, las estimaciones más adecuadas son PTF2 y PTFA2, para el primero y los dos últimos periodos, respectivamente. Del mismo modo, si se consideraran las alternativas PTF1 y PTFA 1, los resultados serían erróneos ya que ocasionaría problemas de regresión espuria y omisión de variables relevantes (Tabla 2).

Para el periodo 1960-1999, la estimación PTF2 y PTFA2 indica que la PTF es $-1.53 \%$ y $-0.62 \%$ respectivamente, lo cual difiere a lo obtenido por Carranza, Fernández-Baca y Morón (2003) quienes obtuvieron $-0.33 \%$ utilizando la metodología de cointegración de Johansen. Más allá del método usado, la diferencia puede explicarse debido a que dichos autores utilizaron las series monetarias en millones de dólares de 1995, mientras que en el presente estudio se utilizó la misma información pero en millones de nuevos soles de 1994. Asimismo, Carranza, Fernández-Baca y Morón (2003) asumieron que la tasa de depreciación es $2.5 \%$ y que el aporte del stock de capital al producto es de $33 \%$, la cual difiere con la tasa de depreciación del presente estudio (5\%).

En síntesis, los resultados ${ }^{6}$ evidencian lo que ocurrió en la sociedad peruana durante los años 80 's, donde hubo violencia terrorista, inestabilidad política y caos macroeconómico

6 Los estudios de Vega - Centeno (1997), Seminario y Beltrán (1998) e IPE (2003) no se pudieron comparar puesto que ellos calcularon la tasa de crecimiento de la PTF por decenios para el periodo 1950-1995. 


\section{Tabla 2. Comparación de resultados ${ }^{2 /}$ de la PTFA y PTF (\%) por periodos}

\begin{tabular}{|c|c|c|c|c|c|c|c|c|c|}
\hline $\begin{array}{c}1960- \\
1999\end{array}$ & Variables & $\mathbf{s}_{\mathrm{K}}$ & $\mathbf{s}_{\mathrm{L}}$ & $\mathbf{s}_{\mathrm{H}}$ & $s_{z}$ & $b_{1}$ & $b_{2}$ & PTF & PTFA \\
\hline PTF1 & $\mathrm{k}, \mathrm{l}, \mathrm{h}$ & $\begin{array}{l}0.8971 \\
{[0.00]}\end{array}$ & $\begin{array}{c}0.1642 \\
{[0.00]}\end{array}$ & $\begin{array}{l}1.3012 \\
{[0.20]}\end{array}$ & & $\begin{array}{c}21.0914 \\
{[0.04]}\end{array}$ & & $-3.46 \%$ & - \\
\hline PTF2 & $k, \mathrm{l}$ & $\begin{array}{l}0.9431 \\
{[0.00]}\end{array}$ & $\begin{array}{c}0.0569 \\
{[0.00]}\end{array}$ & - & - & $\begin{array}{c}-0.2693 \\
{[0.00]}\end{array}$ & - & $-1.53 \%$ & - \\
\hline PTFA1 & $\mathrm{k}, \mathrm{I}, \mathrm{h}, \mathrm{z}$ & $\begin{array}{c}0.6491 \\
{[0.00]}\end{array}$ & $\begin{array}{c}0.4788 \\
{[0.32]}\end{array}$ & $\begin{array}{c}-0.5104 \\
{[0.30]}\end{array}$ & $\begin{array}{c}0.3825 \\
{[0.00]}\end{array}$ & $\begin{array}{c}0.0020 \\
{[0.77]}\end{array}$ & $\begin{array}{c}0.0021 \\
{[0.00]}\end{array}$ & - & $0.17 \%$ \\
\hline PTFA2 & $\mathrm{k}, \mathrm{l}, \mathrm{z}$ & $\begin{array}{c}0.6434 \\
{[0.00]}\end{array}$ & $\begin{array}{l}-0.011 \\
{[0.41]}\end{array}$ & & $\begin{array}{c}0.3674 \\
{[0.00]}\end{array}$ & $\begin{array}{c}0.0174 \\
{[0.00]}\end{array}$ & $\begin{array}{c}0.0173 \\
{[0.00]}\end{array}$ & - & $-0.62 \%$ \\
\hline $\begin{array}{l}1993- \\
2009\end{array}$ & Variables & $\mathbf{s}_{\mathrm{K}}$ & $s_{L}$ & $\mathbf{s}_{\mathrm{H}}$ & $s_{z}$ & $b_{1}$ & $b_{2}$ & PTF & PTFA \\
\hline PTF1 & $\mathrm{k}, \mathrm{l}, \mathrm{h}$ & $\begin{array}{c}0.8236 \\
{[0.00]}\end{array}$ & $\begin{array}{c}-0.4412 \\
{[0.87]}\end{array}$ & $\begin{array}{c}0.6176 \\
{[0.04]}\end{array}$ & - & $\begin{array}{c}-0.0088 \\
{[0.00]}\end{array}$ & - & $0.39 \%$ & - \\
\hline PTF2 & $k, l$ & $\begin{array}{c}0.8575 \\
{[0.00]}\end{array}$ & $\begin{array}{c}0.1425 \\
{[0.00]}\end{array}$ & - & - & $\begin{array}{c}0.0667 \\
{[0.00]}\end{array}$ & - & $0.95 \%$ & - \\
\hline PTFA1 & $\mathrm{k}, \mathrm{l}, \mathrm{h}, \mathrm{z}$ & $\begin{array}{c}0.5942 \\
{[0.00]}\end{array}$ & $\begin{array}{c}-0.3113 \\
{[0.40]}\end{array}$ & $\begin{array}{c}0.4041 \\
{[0.31]}\end{array}$ & $\begin{array}{c}0.3130 \\
{[0.07]}\end{array}$ & $\begin{array}{c}5.6602 \\
{[0.99]}\end{array}$ & $\begin{array}{c}5.6569 \\
{[0.00]}\end{array}$ & - & $0.86 \%$ \\
\hline PTFA2 & $k, I, z$ & $\begin{array}{c}0.5917 \\
{[0.00]}\end{array}$ & $\begin{array}{c}0.0632 \\
{[0.14]}\end{array}$ & - & $\begin{array}{c}0.3451 \\
{[0.05]}\end{array}$ & $\begin{array}{c}0.0337 \\
{[0.00]}\end{array}$ & $\begin{array}{c}0.0237 \\
{[0.00]}\end{array}$ & - & $1.03 \%$ \\
\hline $\begin{array}{l}1960- \\
2009\end{array}$ & Variables & $\mathbf{s}_{\mathrm{K}}$ & $s_{L}$ & $\mathbf{s}_{\mathrm{H}}$ & $s_{z}$ & $b_{1}$ & $b_{2}$ & PTF & PTFA \\
\hline PTF1 & $\mathrm{k}, \mathrm{l}, \mathrm{h}$ & $\begin{array}{c}0.9704 \\
{[0.00]}\end{array}$ & $\begin{array}{c}0.9359 \\
{[0.50]}\end{array}$ & $\begin{array}{c}-0.9063 \\
{[0.00]}\end{array}$ & - & $\begin{array}{c}0.0014 \\
{[0.00]}\end{array}$ & - & $0.13 \%$ & - \\
\hline PTF2 & $k, 1$ & $\begin{array}{c}0.9368 \\
{[0.00]}\end{array}$ & $\begin{array}{c}0.0632 \\
{[0.00]}\end{array}$ & - & - & $\begin{array}{c}-0.1739 \\
{[0.00]}\end{array}$ & - & $-1.09 \%$ & - \\
\hline PTFA1 & $\mathrm{k}, \mathrm{l}, \mathrm{h}, \mathrm{z}$ & $\begin{array}{c}0.6236 \\
{[0.00]}\end{array}$ & $\begin{array}{c}1.1740 \\
{[0.13]}\end{array}$ & $\begin{array}{c}-1.2393 \\
{[0.12]}\end{array}$ & $\begin{array}{c}0.4416 \\
{[0.00]}\end{array}$ & $\begin{array}{c}0.0099 \\
{[0.00]}\end{array}$ & $\begin{array}{c}0.0100 \\
{[0.00]}\end{array}$ & - & $1.60 \%$ \\
\hline PTFA2 & $\mathrm{k}, \mathrm{l}, \mathrm{z}$ & $\begin{array}{c}0.5945 \\
{[0.00]}\end{array}$ & $\begin{array}{c}-0.0153 \\
{[0.39]}\end{array}$ & - & $\begin{array}{c}0.4208 \\
{[0.00]}\end{array}$ & $\begin{array}{c}-0.0029 \\
{[0.84]}\end{array}$ & $\begin{array}{c}-0.0027 \\
{[0.00]}\end{array}$ & - & $-0.11 \%$ \\
\hline
\end{tabular}

2/ Los valores en corchete representan el p-value. Detalles en Anexo 4.

Fuente: Elaboración propia.

que conllevó a un uso ineficiente de los recursos. En efecto, Blyde y Fernández Arias (2005) estimaron que el crecimiento en la PTF del Perú durante el período 1960-99 fue el más bajo de Sudamérica, con excepción de Venezuela. Además, dichos autores descubrieron que al ajustar por el nivel de desarrollo, de los 21 países de América Latina y el Caribe analizados, el Perú ocupó el último lugar en términos del nivel de la PTF.
Considerando el periodo 1993-2009, donde no hubo inestabilidad económica y social, la PTF obtenida es $1.03 \%$. Este incremento se explica por el dinamismo en la inversión, mayores puestos de trabajo, incremento de las emisiones de $\mathrm{CO}_{2}$ y el hecho que las políticas públicas estuvieron orientadas a lograr la reforma del Estado y la organización de la producción en la economía. 
Si bien se ha incrementado la PTF para el último periodo (debido al extraordinario crecimiento peruano de la última década), este resultado aún sigue siendo reducido. La explicación es que actualmente el $68 \%$ de la PEA trabaja en empresas de no más de cinco trabajadores, en condiciones técnicas y productivas inferiores al promedio latinoamericano. Asimismo, los estándares tecnológicos y de productividad todavía no muestran una propensión suficientemente enérgica hacia la innovación y la competitividad con mayor valor agregado (CEPLAN, 2011).

Otro gran problema en el Perú es el subempleo. Los datos disponibles muestran que en el año 1998 el subempleo afectaba al 49.5\% de la PEA y en el año 2009, la situación había mejorado debido a que el empleo adecuado aumentó a 51.6\%, mientras que el desempleo y subempleo disminuyeron en $2 \%$ y $5 \%$, respectivamente. Actualmente, hay escaso apoyo del gobierno que se complemente con las inversiones en investigación y desarrollo en las universidades y centros de investigación.

Además, el impulso a la innovación en las Medianas y Pequeñas Empresas (MYPE) es bajo y son pocas las asociaciones universidad-empresa, por lo que la duración de crecimiento y la vida útil de aquellas es muy corta. Así, el sector emprendedor no aprovecha las limitadas oportunidades relacionadas con la investigación y el desarrollo, lo que genera insuficiente innovación tecnológica y una menor competitividad del sector en la región. Las productividades muy disímiles impiden las relaciones inter-empresariales y limitan a su vez la sustentación de más empleos produc- tivos, limitando a la mano de obra a bajos ingresos o al subempleo (CEPLAN, 2011).

Por último, si se analizara todo el periodo de estudio (1960-2009), la PTF es -0.11\%.Una posible explicación es que si bien hubo un crecimiento de la PTF durante 1993-2009, este resultado fue opacado principalmente por los hechos ocurridosen los años 1970-1992, donde la productividad fue la menor de América Latina. Asimismo, el aporte negativo de la mano de obra sobre el producto indicaría que si bien existía mano de obra adecuadamente empleada, es decir, tenia condiciones laborales que les permitía satisfacer sus necesidades basicas, la bajas tasas de crecimiento del producto en los ochenta no permitieron sostener la masa laboral (presentándose así la etapa de los rendimientos negativos).

\section{CONCLUSIONES Y RECOMENDACIONES}

De acuerdo a la evidencia empírica desarrollada en el presente estudio, se corrobora que las emisiones de $\mathrm{CO}_{2^{\prime}}$ (proxy de la variable ambiental), explican el producto durante el periodo 1960-2009, excepto para el subperiodo 1960-1990, en el que la relación fue inversa. Por lo tanto, es importante tener en cuenta que la inclusión de la variable ambiental como factor del producto puede variar dependiendo del país y periodo en análisis. En consecuencia, realizar la prueba de causalidad entre ambas variables es necesario en cualquier estudio sobre el crecimiento económico. 
De otro lado, para el periodo $1960-2009$, se demuestra que la PTF es $-0.11 \%$ incluyendo como inputs al stock de capital artificial, mano de obra y las emisiones de $\mathrm{CO}_{2}$. Este resultado fue superior a la PTF medida solo con factores tradicionales (-1.09\%). Por lo tanto, si no se incluye la variable ambiental como input, la PTF obtenida será distorsionada.

Cuando se incluye la variable ambiental, los resultados de las participaciones de los factores en el producto difieren sustancialmente durante los periodos 1993-2009 y 1960-2009. La explicación puede deberse a que más allá de utilizar distintas metodologías o utilizar las series monetarias en diferentes unidades de medida, posiblemente al no incluir la variable ambiental en el análisis, los resultados de las contribuciones de los inputs en el producto serán sesgados.

Sobre las emisiones de $\mathrm{CO}_{2}$ una consideración sería realizar investigaciones utilizando otros contaminantes a fin de contrastar las posibles variaciones en los resultados obtenidos en este estudio. Asimismo, sería interesante desarrollar el modelo neoclásico incorporando el stock de capital natural, aunque esto podría resultar ser difícil debido a problemas de agregación o por no disponer de información histórica.

Por último, si el Perú quiere salir del subdesarrollo y perseverar una senda de crecimiento alto y sostenido, tendrá que focalizar esfuerzos en lograr mejoras sustanciales en sus niveles de productividad. Si bien a nivel macroeconómico, la inversión en capital físico y humano resulta ser una condición necesaria para el crecimiento sostenido, no es suficiente (Alarco, 2011). Por ello, el gobierno debe enfocarse en tener una mejor eficiencia en el uso de los recursos, como también prestar especial atención al efecto perjudicial de la informalidad sobre los incentivos y mecanismos para una mayor productividad sectorial y empresarial.

\section{AGRADECIMIENTOS:}

Los autores desean agradecer a los dos dictaminadores anónimos por sus observaciones constructivas así como a Roberto Escalante y Juan Pichihua por sus valiosos comentarios en una versión preliminar de este estudio. Como es costumbre, cualquier error u omisión es responsabilidad exclusiva de los autores. 


\section{REFERENCIAS BIBLIOGRÁFICAS}

Aghion, P.; y Howitt, P. (1992). "A Model of Growth through Creative Destruction", Econometrica, Econometric Society, vol. 60(2), pp. 323-51.

Alarco, G. (2011). Competitividad y DesarroIlo: Evolución y Perspectivas Recientes. CENTRUM Católica, Centro de Negocios de la Pontificia Universidad Católica del Perú.

Barro, R., Lee, J. (2000). "International Data on Educational Attainmed: Updates and Implications, National Bureau of Economic Research Working Papers, 7911.

BCRP (2011). Series Estadisticas Anuales del Banco Central de Reserva del Perú (BCRP). Disponible en http://estadisticas.bcrp.gob.pe/.

Becker, R. (1982). "Intergenerational equity: The capital environment trade-off", Journal of Environmental Economics and Management 9: 165-185.

Blyde, J., and Fernández-Arias, E.(2005). “Why Latin America is Falling Behind." In: E. Fernández-Arias, R. Manuelli and J.S. Blyde, editors. Sources of Growth in Latin America: What is Missing? Washington, DC, United States: Inter-American Development Bank.

Brock, W. (1973). Transitional impacts of environmental policy in an endogenous growth model. International Economic Reviews, 37, 861-893.
Carranza, E., Fernández-Baca, J. y Morón, E. (2003). "Markets, Government, and the Sources of Growth in Peru", Universidad delPacífico, Departamento de Economía, mimeografiado.

CEPLAN (2011). Plan Bicentenario: El Perú hacia el 2021. Centro Nacional de Planeamiento Estratégico. Disponible en www.ceplan.gob.pe

Chimeli, A., Braden, J. (2005). Total factor productivity and the environmental Kuznets curve. Journal of EnvironmentalEconomics and Management, 49, 366-380.

CONAM (2001). Comunicación Nacional del Perú a la Convención de Naciones Unidas sobre el Cambio Climático. Junio 2001.

Coondoo, D., Dinda, S. (2002). Causality between income and emission: a country group-specific econometric analysis. Ecological Economics, 40(3), 351-367.

Dasgupta, P., y Heal, G. (1979). Economic Theory and Exhaustible Resources, Cambridge Economic Handbook, Cambridge.

Dasgupta, P., Mäler, K. (2000). Net national product, wealth and social well-being.Environmental and Development Economics, 5(12), 69-93.

De Bruyn, S., Van den Berghand, J., Opschoor, B. (1998). Economic Growth and Emissions: Reconsidering the Empirical Basis of Environmental Kuznets Curves. Ecological Economics, 25(2), 161-175. 
Denison, E. (1962). The Sources of Economic Growth in the United States and the Alternative Before, Us. Washington DC: Committee for Economic Development.

Ekins, P. (1997). The Kuznets curve for the environment and economic growth: examining the evidence. Environment and Planning, 29(5), 805-830.

Georgescu-Roegen, N. (1975). Energy and economic myths. Southern Economic Journal, 41(3), 347-381.

Granger, C. (1988).Some recent developments in the concept of causality.Journal of Econometrics, 39(1-2), 199-211.

Green, W. (1990)."Econometric Analysis", Macmillan, New York, Second Edition.

Grossman, G., Helpman, E. (1991). Innovation and growth in the global economy, Cambridge, MA, MIT Press, Cambridge.

Gruver, G.W. (1976). "Optimal investment in pollution control capital in a neoclassical growth context", Journal of Environmental Economics and Management, 3, 165-177.

Harbaugh, W., Levinson A., Wilson, D. (2002). Reexamining the Empirical Evidence for an Environmental Kuznets Curve. The Review of Economics and Statistics, 84(3), 541-551.

INEI (2010a). Perú: Evolución de los Indicadores de Empleo e Ingresos por Departamentos, 2001-2009. Dirección Nacional de Cuentas
Nacionales. Documento no publicado.

INEI (2010b). Perú: Anuario de Estadísticas Ambientales 2010. Disponible en www.inei. gob.pe. Fecha de actualización: 2010.

IPE (2003). La brecha en infraestructura, servicios públicos, productividad y crecimiento en el Perú. En J. Coronado (Ed.), Asociación de Empresas Privadas de Servicios Públicos (pp. 19-71), Lima: Instituto Peruano de Economía.

Jiménez, F. (2011).Producto potencial, fuentes del crecimiento y productividad en la economía peruana (1950-2008). El Trimestre Económico, 78 (4), 913-940.

Kalaitzidakis, P., Mamuneas, T., Stengos, T. (2006).The contribution of Pollution to Productivity Growth.Working Paper.

Keeler, E., M. Spence and R. Zeckhauser (1971).“The optimal control of pollution", Journal of Economic Theory 4: 19-34.

Menyah, K., Wolde-Rufael, Y. (2010).Energy consumption, pollutant emissions and economic growth in South Africa.EnergyEconomics, 32(6), 1374-1382.

MINAM (2010). Huella de Carbono del Ministerio del Ambiente. Informe Final. Disponible en www.minam.gob.pe

MINEM (2010). Balance Nacional de Energía 2009. Dirección General de Eficiencia Energética. Disponible en www.minem.gob.pe 
Mundlak, Y. (1996). Production Function Estimation: Reviving the Primal. Econometrica, 64(2), 431-438.

Romer, P. (1987). Growth based on increasing returns due to specialization, The American Economic Review, 77(2), 56-62.

Seminario, B. y Beltrán, A. (1998). Crecimiento económico en el Perú: 1896-1995. Nuevas evidencias estadísticas. Centro de Investigación de la Universidad del Pacifico (CIUP).

Seminario, B. (2004).Metodología para la elaboración de Proyecciones Macroeconómicas: Perú 2004-2025. Versión Preliminar. Centro de Investigación de la Universidad del Pacifico (CIUP).

Seminario, B., Rodríguez, M., Zuloeta, J. (2008). Métodos alternativos para la estimación del PBI potencial 1950-2007. Centro de Investigación de la Universidad del Pacifico (CIUP), Documento de trabajo, http://www.up.edu. pe/ciup/SiteAssets/Lists/JER_Jerarquia/EditForm/20090112144424_DD\%2007-20\%20 v8_final1.pdf.

Solow, R. (1957). Technical Change and the Aggregate Production Function.Review of Economics and Statistics, 39(3), 312-320.

Stern, D., Common, N.; y Barbier, E. (1996).“Economic growth and environmental degradation: the environmental Kuznets curve and sustainable development", World Development, 24, 1151-1160.
Stern, D. (2004). "The Rise and Fall of the Environmental Kuznets Curve". World Development, 32(8), 1419-1439.

Tzouvelekas E., Vouvaki, D. and Xepapadeas, A. (2007). "Total Factor Productivity Growth and the Environment: A Case for Green Growth Accounting". CCMP - Climate Change Modelling and Policy, Nota Di-Lavoro, 38,http:// www.feem.it/userfiles/attach/Publication/ NDL2007/NDL2007-038.pdf.

World Bank (2011). World Development Indicators, p.435. Disponible en: http://www. preventionweb.net/english/professional/publications/v.php?id=19618

Xepapadeas, A. (2003). Economic Growth and the Environment.University of Crete, Department of Economics, Rethymno, Greece.

Xepapadeas, A. (2005). Economic Growth and the Environment.Handbook of Environmental Economics, Vol. 3, Elsevier Publishers.

Yamada, G. (2006). "Retornos a la educación superior en el mercado laboral. ¿Vale la pena el esfuerzo?", Documento de Discusión núm. 6, CIUP.

Yamada, G. (2007). "Retornos a la educación superior en el mercado laboral: ¿Vale la pena el esfuerzo?. Universidad del Pacifico-Centro de Investigación y CIES, Documento de trabajo, (78), http://cies.org.pe/files/documents/investigaciones/educacion/retornos-a-la-educacion-superior-en-el-mercado-laboral.pdf.

Vega-Centeno, M. (1997). “Inestabilidad e in- 
suficiencia del crecimiento: el desempeño de la economía peruana 1950-1996". Pontificia
Universidad Católica del Perú, Revista Economía, 39-40, 11-62.

\section{ANEXOS}

\section{Anexo 1}

Para la obtención dela PTF, la función de producción tradicional se define como:

$$
Y=F(K, E)=F(K, A L)
$$

La igualdad anterior se puede expresar de la siguiente manera:

$$
\mathrm{Y}=K^{S_{K}}(A L)^{S_{L}}(A .2)
$$

Aplicando logaritmos se obtiene:

$$
\begin{aligned}
\log Y & =\log K^{S_{K}}+\log A^{S_{L}}+\log L^{S_{L}} \\
\log Y & =S_{K} \log K+S_{L} \log A+S_{L} \log L \\
\frac{\partial \log Y}{\partial t} & =\frac{\partial\left(s_{K} \log K+s_{L} \log A+s_{L} \log L\right)}{\partial t} \\
\frac{\dot{Y}}{Y} & =s_{K}\left(\frac{\dot{K}}{K}\right)+s_{L}\left(\frac{\dot{A}}{A}\right)+s_{L}\left(\frac{\dot{L}}{L}\right)
\end{aligned}
$$

Por tanto, la PTF se define como:

$$
g_{S}=s_{L}\left(\frac{\dot{A}}{A}\right)=\frac{\dot{Y}}{Y}-s_{K}\left(\frac{\dot{K}}{K}\right)-s_{L}\left(\frac{\dot{L}}{L}\right)
$$

Y luego aplicando logaritmos:

$$
\begin{gathered}
g_{S}=s_{L}(\log A)=\log Y-s_{K} \log K-\left(1-s_{K}\right) \log L \\
g_{S}=s_{L}(\log A)=\log Y-s_{K} \log K-\log L+s_{K} \log L \\
g_{S}=s_{L}(\log A)=\log Y-\log L-s_{K}(\log K-\log L)
\end{gathered}
$$




$$
\begin{gathered}
g_{S}=s_{L}(\log A)=\log \left(\frac{Y}{L}\right)-s_{K}\left[\log \left(\frac{K}{L}\right)\right] \\
g_{S}=s_{L}\left(\frac{\partial \log A}{\partial t}\right)=\frac{\partial \log \left(\frac{Y}{L}\right)}{\partial t}-s_{K}\left[\frac{\partial \log \left(\frac{K}{L}\right)}{\partial t}\right]
\end{gathered}
$$

Asumiendo retornos constantes a escala ( $s_{K}+s_{L}=1$ ), se obtiene:

$$
g_{S}=s_{L}\left(\frac{\dot{A}}{A}\right)=\frac{\dot{Y}}{Y}-s_{K}\left(\frac{\dot{k}}{k}\right)
$$

\section{Anexo 2}

Ahora, incluyendo el factor ambiental, la función de producción estaría definida como:

$$
Y=F(K, H, E, X)(A .14)
$$

Aplicando logaritmos:

$$
\log Y=\log K^{s_{K}}+\log H^{s_{H}}+\log A^{s_{L}}+\log L^{S_{L}}+\log B^{s_{Z}}+\log Z^{s_{Z}}
$$

Diferenciando lo anterior respecto del tiempo, se obtiene

$$
\frac{\partial \log Y}{\partial t}=\frac{s_{K} \partial \log K}{\partial t}+\frac{s_{H} \partial \log H}{\partial t}+\frac{s_{L} \partial \log A}{\partial t}+\frac{s_{L} \partial \log L}{\partial t}+\frac{s_{Z} \partial \log B}{\partial t}+\frac{s_{Z} \partial \log Z}{\partial t}
$$

Luego, definiendo a $s_{j}, j=K, H, L, Z$ como las elasticidades de los inputs hacia el producto y si la PTFA $(\gamma)$ está dado por (A.17):

$$
\gamma=s_{L}\left(\frac{\dot{A}}{A}\right)+s_{L}\left(\frac{\dot{B}}{B}\right)=\left(\frac{\dot{Y}}{Y}\right)-s_{k}\left(\frac{\dot{K}}{K}\right)-s_{H}\left(\frac{\dot{H}}{H}\right)-s_{L}\left(\frac{\dot{L}}{L}\right)-s_{Z}\left(\frac{\dot{Z}}{Z}\right)
$$

Bajo retornos constantes a escala $\left(s_{K}+s_{H}+s_{Z} s_{L}=1\right.$ ó $\left.s_{L}=1 s_{K}+s_{H}+s_{Z}\right)$, se tiene que:

$$
\begin{gathered}
\gamma=s_{L}\left(\frac{\dot{A}}{A}\right)+s_{L}\left(\frac{\dot{B}}{B}\right)=\left(\frac{\dot{Y}}{Y}\right)-s_{k}\left(\frac{\dot{K}}{K}\right)-s_{H}\left(\frac{\dot{H}}{H}\right)-\left[1-s_{K}-s_{H}-s_{Z}\right]\left(\frac{\dot{L}}{L}\right)-s_{Z}\left(\frac{\dot{Z}}{Z}\right) \\
\frac{\dot{Y}}{Y}-\frac{\dot{L}}{L}=\gamma+s_{k}\left(\frac{\dot{K}}{K}-\frac{\dot{L}}{L}\right)+s_{H}\left(\frac{\dot{H}}{H}-\frac{\dot{L}}{L}\right)+s_{Z}\left(\frac{\dot{Z}}{Z}-\frac{\dot{L}}{L}\right) \\
\frac{\partial \log \left(\frac{Y}{L}\right)}{\partial t}=\gamma+s_{k}\left[\frac{\partial \log \left(\frac{K}{L}\right)}{\partial t}\right]+s_{H}\left[\frac{\partial \log \left(\frac{H}{L}\right)}{\partial t}\right]+s_{Z}\left[\frac{\partial \log \left(\frac{Z}{L}\right)}{\partial t}\right]
\end{gathered}
$$


La expresión (19)se obtiene de (A.23):

$$
\frac{\dot{y}}{y}=\gamma+s_{k} \frac{\dot{k}}{k}+s_{H} \frac{\dot{h}}{h}+s_{Z} \frac{\dot{z}}{z} \quad(A .23)
$$

\section{Anexo 3. Matriz de correlación de las variables explicativas}

\begin{tabular}{|c|c|c|c|c|}
\hline $\mathbf{V}$ & $\mathbf{K}$ & $\mathbf{H}$ & $\mathbf{L}$ & $\mathbf{Z}$ \\
\hline K & 1.000000 & 0.975955 & 0.982044 & 0.651099 \\
H & 0.975955 & 1.000000 & 0.997154 & 0.600224 \\
L & 0.982044 & 0.997154 & 1.000000 & 0.610070 \\
Z & 0.651099 & 0.600224 & 0.610070 & 1.000000 \\
\hline
\end{tabular}

Fuente: Elaboración propia

\begin{tabular}{|c|c|c|c|c|c|}
\hline \multicolumn{6}{|c|}{ Anexo 4. Test estadísticos en los modelos PTFA y PTF } \\
\hline 1960-1999 & Variables & $\begin{array}{c}\mathbf{R}^{2} \\
\text { ajustado }\end{array}$ & $\begin{array}{c}\text { Prob. } \\
\text { Prueba F }\end{array}$ & $\begin{array}{c}\text { Prob. } \\
\text { Test BPG }{ }^{3 /}\end{array}$ & $\begin{array}{c}\text { Prob. } \\
\text { Test LM }{ }^{4 /}\end{array}$ \\
\hline PTF1 & $\mathrm{k}, \mathrm{I}, \mathrm{h}$ & 0.66 & 0.00 & 0.31 & 0.00 \\
\hline PTF2 & $k, \mathrm{l}$ & 0.65 & 0.00 & 0.78 & 0.00 \\
\hline PTFA1 & $\mathrm{k}, \mathrm{l}, \mathrm{h}, \mathrm{z}$ & 0.88 & 0.00 & 0.00 & 0.00 \\
\hline PTFA2 & $\mathrm{k}, \mathrm{l}, \mathrm{z}$ & 0.88 & 0.00 & 0.00 & 0.00 \\
\hline 1993-2009 & Variables & $\begin{array}{c}\mathbf{R}^{2} \\
\text { ajustado }\end{array}$ & $\begin{array}{c}\text { Prob. } \\
\text { Prueba F }\end{array}$ & $\begin{array}{c}\text { Prob. } \\
\text { Test BPG* }\end{array}$ & $\begin{array}{c}\text { Prob. } \\
\text { Test LM }^{\star *}\end{array}$ \\
\hline PTF1 & $\mathrm{k}, \mathrm{l}, \mathrm{h}$ & 0.63 & 0.00 & 0.57 & 0.04 \\
\hline PTF2 & $\mathrm{k}, \mathrm{l}$ & 0.59 & 0.00 & 0.97 & 0.00 \\
\hline PTFA1 & $\mathrm{k}, \mathrm{I}, \mathrm{h}, \mathrm{z}$ & 0.81 & 0.00 & 0.42 & 0.05 \\
\hline PTFA2 & $\mathrm{k}, \mathrm{l}, \mathrm{z}$ & 0.81 & 0.00 & 0.72 & 0.00 \\
\hline 1960-2009 & Variables & $\begin{array}{c}\mathbf{R}^{2} \\
\text { ajustado }\end{array}$ & $\begin{array}{c}\text { Prob. } \\
\text { Prueba F }\end{array}$ & $\begin{array}{c}\text { Prob. } \\
\text { Test BPG }\end{array}$ & $\begin{array}{c}\text { Prob. } \\
\text { Test LM }{ }^{* *}\end{array}$ \\
\hline PTF1 & $\mathrm{k}, \mathrm{l}, \mathrm{h}$ & 0.61 & 0.00 & 0.20 & 0.00 \\
\hline PTF2 & $\mathrm{k}, \mathrm{l}$ & 0.60 & 0.00 & 0.19 & 0.00 \\
\hline PTFA1 & $\mathrm{k}, \mathrm{l}, \mathrm{h}, \mathrm{z}$ & 0.87 & 0.00 & 0.00 & 0.00 \\
\hline PTFA2 & $\mathrm{k}, \mathrm{l}, \mathrm{z}$ & 0.85 & 0.00 & 0.00 & 0.00 \\
\hline & & $\begin{array}{r}\text { 3/ Test de } \\
\text { 4/ Test de Br } \\
\text { Fuent }\end{array}$ & $\begin{array}{l}\text {-Pagan-God } \\
\text { odfrey (2 re } \\
\text { ración propia }\end{array}$ & & \\
\hline
\end{tabular}

